\title{
Evaluation of a 55-gene classifier as a prognostic biomarker for adjuvant chemotherapy in stage III colon cancer patients
}

\author{
Eiji Oki ${ }^{*}$, Eiji Shinto ${ }^{2}$, Mototsugu Shimokawa $^{3}$, Shigeki Yamaguchi ${ }^{4}$, Megumi Ishiguro $^{5}$, Seiji Hasegawa ${ }^{6}$, \\ Yasumasa Takii ${ }^{7}$, Hideyuki Ishida ${ }^{8}$, Tetsuya Kusumoto ${ }^{9}$, Masaru Morita ${ }^{10}$, Naohiro Tomita ${ }^{11}$, Manabu Shiozawa ${ }^{12}$, \\ Masafumi Tanaka ${ }^{13}$, Heita Ozawa ${ }^{14}$, Yojiro Hashiguchi ${ }^{15}$, Shinobu Ohnuma ${ }^{16}$, Sachiyo Tada ${ }^{17}$, \\ Tomoko Matsushima ${ }^{17}$ and Kazuo Hase ${ }^{2}$
}

\begin{abstract}
Background: Adjuvant chemotherapy reduces the risk of recurrence of stage III colon cancer (CC). However, more effective prognostic and predictive biomarkers are needed for better treatment stratification of affected patients. Here, we constructed a 55-gene classifier (55GC) and investigated its utility for classifying patients with stage III CC.

Methods: We retrospectively identified patients aged 20-79years, with stage III CC, who received adjuvant chemotherapy with or without oxaliplatin, between the years 2009 and 2012.

Results: Among 938 eligible patients, 203 and 201 patients who received adjuvant chemotherapy with and without oxaliplatin, respectively, were selected by propensity score matching. Of these, 95 patients from each group were analyzed, and their 5-year relapse-free survival (RFS) rates with and without oxaliplatin were 73.7 and $77.1 \%$, respectively. The hazard ratios for 5-year RFS following adjuvant chemotherapy (fluoropyrimidine), with and without oxaliplatin, were $1.241(95 \% \mathrm{Cl}, 0.465-3.308 ; P=0.67)$ and $0.791(95 \% \mathrm{Cl}, 0.329-1.901 ; P=0.60)$, respectively. Stratification using the 55GC revealed that 52 (27.3\%), 78 (41.1\%), and 60 (31.6\%) patients had microsatellite instability (MSI)-like, chromosomal instability (CIN)-like, and stromal subtypes, respectively. The 5 -year RFS rates were 84.3 and $72.0 \%$ in patients treated with and without oxaliplatin, respectively, for the MSI-like subtype (HR, $0.495 ; 95 \% \mathrm{Cl}, 0.145-1.692 ; P=0.25)$. No differences in RFS rates were noted in the CIN-like or stromal subtypes. Stratification by cancer sidedness for each subtype showed improved RFS only in patients with left-sided primary cancer treated with oxaliplatin for the MSI-like subtype $(P=0.007)$. The 5 -year RFS rates of the MSI-like subtype in left-sided cancer patients were 100 and $53.9 \%$ with and without oxaliplatin, respectively.
\end{abstract}

Conclusions: Subclassification using 55GC and tumor sidedness revealed increased RFS in patients within the MSI-like subtype with stage III left-sided CC treated with fluoropyrimidine and oxaliplatin compared to those treated without oxaliplatin. However, the predictive power of 55GC subtyping alone did not reach statistical significance in this cohort, warranting larger prospective studies.

\footnotetext{
*Correspondence: okieiji@surg2.med.kyushu-u.ac.jp

${ }^{1}$ Department of Surgery and Science, Graduate School of Medical

Sciences, Kyushu University, 3-1-1, Maidashi, Higashi-ku,

Fukuoka 812-8582, Japan

Full list of author information is available at the end of the article
}

(c) The Author(s) 2021. Open Access This article is licensed under a Creative Commons Attribution 4.0 International License, which permits use, sharing, adaptation, distribution and reproduction in any medium or format, as long as you give appropriate credit to the original author(s) and the source, provide a link to the Creative Commons licence, and indicate if changes were made. The images or other third party material in this article are included in the article's Creative Commons licence, unless indicated otherwise in a credit line to the material. If material is not included in the article's Creative Commons licence and your intended use is not permitted by statutory regulation or exceeds the permitted use, you will need to obtain permission directly from the copyright holder. To view a copy of this licence, visit http://creativecommons.org/licenses/by/4.0/. The Creative Commons Public Domain Dedication waiver (http://creativeco mmons.org/publicdomain/zero/1.0/) applies to the data made available in this article, unless otherwise stated in a credit line to the data. 
Trial registration: The study protocol was registered in the University Hospital Medical Education Network (UMIN) clinical trial registry (UMIN study ID: 000023879).

Keywords: Colon cancer, Predictive, Adjuvant chemotherapy, Oxaliplatin, Subtyping

\section{Background}

Colorectal cancer remains one of the most common causes of cancer-related mortality worldwide [1]. Adjuvant chemotherapy in stage III colon cancer (CC) after curative intent resection prolongs survival and reduces the risk of tumor recurrence [2]. Pivotal trials have shown superior outcomes for fluoropyrimidine in combination with oxaliplatin compared with fluoropyrimidine alone in most patient populations; however, the evidence is less well established in elderly patients. Emerging data have also led to a debate over the optimal duration of chemotherapy, specifically in the context of increased toxicity [3]. Furthermore, subclassification of stage III CC is an ongoing process based on accumulating patient survival data and features of cancer presentation [4]. Therefore, better prognostic and predictive biomarkers are required to stratify patients for adjuvant therapies based on chemotherapy regimen and duration.

The consensus molecular subtype (CMS) is a robust classification system of colorectal cancer types based on over 600 genes. DNA microarray analysis of CMS can provide a valuable prognostic information [5]. CMS is also potentially predictive, as different subtypes vary in sensitivity to adjuvant chemotherapy. We previously simplified this classification by constructing a 55-gene classifier (55GC), focusing on genes located on the long arms of chromosomes 18 and 20, as well as on stroma-related genes [6]. Using the 55GC, we categorized stage II/III CC into three subtypes with different recurrence rates: "microsatellite instability (MSI)-like," "chromosomal instability (CIN)-like," and "stromal" subtypes and showed a prognostic utility of such a system in a single institutional study. We conducted a validation study using a $\underline{55}$-gene classifier to assess stratification recurrence (55 STAR) risk. Furthermore, 55GC-based subtyping was able to stratify stage II CC recurrence risk in a multi-institutional validation cohort study of 232 patients [7]. To expand on these previous findings, we hypothesized that $55 \mathrm{GC}$ could be utilized to stratify survival of patients with stage III CC who receive adjuvant chemotherapy with or without oxaliplatin.

\section{Methods}

\section{Tissue samples}

We retrospectively identified consecutive patients with stage III colon and rectosigmoid CC aged 20-79years who underwent curative surgery (R0) and received adjuvant chemotherapy with or without oxaliplatin from 15 institutions in Japan between January 1, 2009, and December 31, 2012. Patients who received neoadjuvant treatment, had multiple active cancers, died, or had recurrence within 60 days post-surgery, were excluded from the study. Relevant patient characteristics were recorded. The study protocol was approved by the institutional review boards of Kyushu University (study ID 28-69), National Defense Medical College (study ID 2477), Saitama Medical University International Medical Center (study ID 16-051), Tokyo Medical and Dental University (study ID G2016-007), Saiseikai Yokohamashi Nanbu Hospital (study ID 2017-D21), Niigata Cancer Center Hospital (study ID 796), Saitama Medical University Saitama Medical Center (study ID 1812), National Hospital Organization Kyushu Medical Center (study ID 16C058), National Hospital Organization Kyushu Cancer Center (study ID 2016-48), Hyogo College of Medicine (study ID Hi326), Kanagawa Cancer Center (study ID 2017-8), Takano Hospital (study ID 16-04), Tochigi Cancer Center (study ID A432), Teikyo University School of Medicine (study ID 16-057), Tohoku University (study ID 2016-1-222) and Sysmex Corporation (study ID 2015-71), and was registered in the University Hospital Medical Education Network Clinical Trial Registry (UMIN study ID 000023879). All procedures were carried out in accordance with the relevant guidelines and regulations. Because this study was a retrospective observational study carried out in Japan, informed consent was obtained using the optout/opt-in approach, according to each participating institution's policy (as per Japanese ethical guidelines for an observational study, consent of the family is not required for dead participants). The Consolidated Standards of Reporting Trials (CONSORT) diagram is shown in Fig. 1.

\section{Gene expression analysis}

Formalin-fixed paraffin-embedded (FFPE) primary cancer tissue specimens containing the invasive tumor front with the greatest depth of invasion were collected from each institution, and a single $5-\mu \mathrm{m}$ section was sent to the Takeda Pathology Center (Osaka, Japan) for analysis. Total RNA was extracted from fewer than four unstained $10-\mu \mathrm{m}$ sections for gene expression microarray assay profiling using the RNeasy FFPE Kit (Qiagen, Valencia, CA, 


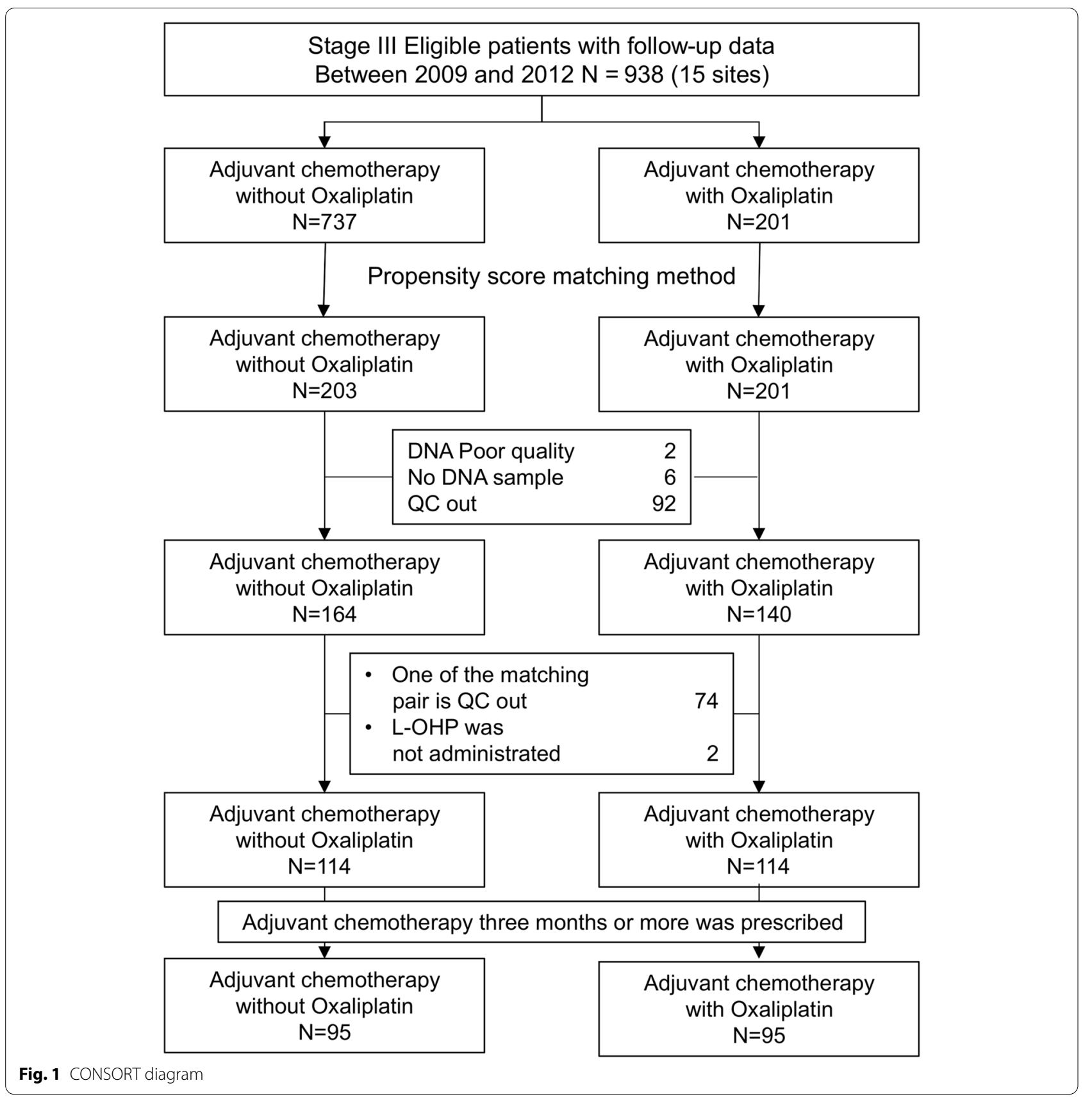

USA). Samples with insufficient RNA quality for microarray analysis were excluded from this cohort. Gene expression data were generated using the Affymetrix GeneChip Human Genome U133 Plus 2.0 Array (Thermo Fisher Scientific; Waltham, MA) and analyzed using the 55GC model as described previously [6]. DNA was extracted using the QIAamp DNA FFPE Tissue Kit (Qiagen). RAS mutations were assessed using the MEBGEN RASKET KIT (Medical \& Biological Laboratories; Nagoya, Japan).

\section{Statistical analyses}

The primary endpoint was RFS, defined as the time from surgery to the first CC recurrence or death from any cause. A propensity score method was used to reduce the selection bias, and a logistic regression model was used to calculate patient propensity scores. Propensity score matching was performed for the number of lymph node metastases, tumor location, sex, and age in a 1:1 ratio using a caliper width 
of 0.1. Demographic characteristics are summarized using contingency tables. The RFS curve was estimated using the Kaplan-Meier method and compared between groups using log-rank tests. HRs and 95\% CIs were calculated using the Cox proportional hazards model. Risk factors for RFS were assessed using a Cox proportional hazards model with a backward elimination method that included known clinicopathological prognostic factors and gene mutations as covariates. Subgroup analysis was performed for age ( $<70$ vs. $\geq 70$ years), sex (male vs. female), carcinoembryonic antigen (<upper limit of normal [ULN] vs. $\geq U L N$ ), tumor location (left vs. right), $\mathrm{T}$ stage (T1-T3 vs. T4), lymph node metastasis (N1 vs. N2-N3), tumor grade (poorly differentiated and mucinous adenocarcinoma vs. tubular adenocarcinoma), vascular invasion (v0 vs. v1-v3), subtype (CIN vs. MSI vs. stromal), and $R A S$ status (wild vs. mutant). Fisher's exact test was used to compare patient characteristics between the groups. $P$-values were two-sided, and statistical significance was set at $P<0.05$. All statistical analyses were performed using the Statistical Analysis System, version 9.4 (SAS Institute, Cary, NC).

\section{Results}

Patient characteristics

Among 938 eligible patients, 203 and 201 individuals receiving adjuvant chemotherapy with and without oxaliplatin, respectively, were selected using propensity score matching. After excluding patients with low-quality specimens and those who had received chemotherapy for $<3$ months, 95 patients from each group were analyzed (Fig. 1). In the overall cohort of 190 patients (Table 1), 98 (51.6\%) patients were men, 146 (76.8\%) were aged < 70years, and 126 (66.3\%) had left-sided tumors. Regarding histopathological characteristics, there were more patients with T4 stage cancer in the cohort treated with oxaliplatin (44/95 patients, $46.3 \%)$ than in the cohort treated without oxaliplatin (34/95 patients, $35.8 \%)(P=0.022)$. In addition, there were more patients with $<12$ resected lymph nodes in the cohort treated with oxaliplatin (15/95 patients, $15.8 \%)$ than in the cohort treated without oxaliplatin (5/95 patients, $5.3 \%$ ) $(P=0.018)$. The 5 -year RFS rates were 73.7 and $77.1 \%$ in patients treated with and without oxaliplatin, respectively (Fig. 2; hazard ratio [HR]: 0.858; 95\% confidence interval [CI]: 0.484-1.522).

Table 1 Patient characteristics

\begin{tabular}{|c|c|c|c|c|c|}
\hline \multirow[t]{2}{*}{ Factors } & & \multirow{2}{*}{$\begin{array}{l}\text { Oxaliplatin }(-)(N=95) \\
\mathrm{n}(\%)\end{array}$} & \multirow{2}{*}{$\begin{array}{l}\text { Oxaliplatin }(+) \\
(N=95) \\
\mathrm{n}(\%)\end{array}$} & \multirow{2}{*}{$\begin{array}{l}\text { Total } \\
(N=190) \\
\mathrm{n}(\%)\end{array}$} & \multirow[t]{2}{*}{$P$ value } \\
\hline & & & & & \\
\hline \multirow[t]{2}{*}{ Sex } & Male & $46(48.4)$ & $52(54.7)$ & $98(51.6)$ & 0.3838 \\
\hline & Female & $49(51.6)$ & $43(45.3)$ & $92(48.4)$ & \\
\hline \multirow[t]{2}{*}{ Age (years) } & $<70$ & $71(74.7)$ & 75 (78.9) & $146(76.8)$ & 0.4915 \\
\hline & $\geq 70$ & $24(25.3)$ & $20(21.1)$ & $44(23.2)$ & \\
\hline \multirow[t]{3}{*}{ CEA } & $<U L N$ & $63(66.3)$ & $50(52.6)$ & $113(59.5)$ & 0.6105 \\
\hline & $\geq U L N$ & $31(32.6)$ & $20(21.1)$ & $51(26.8)$ & \\
\hline & Unknown & $1(1.1)$ & $25(26.3)$ & $26(13.7)$ & \\
\hline \multirow[t]{2}{*}{ Tumor location } & Right side & $31(32.6)$ & $33(34.7)$ & $64(33.7)$ & 0.7588 \\
\hline & Left side & $64(67.4)$ & $62(65.3)$ & $126(66.3)$ & \\
\hline \multirow[t]{2}{*}{ T stage } & $\mathrm{T} 1-\mathrm{T} 3$ & $61(64.2)$ & $51(53.7)$ & $112(58.9)$ & 0.1403 \\
\hline & $\mathrm{T} 4$ & $34(35.8)$ & $44(46.3)$ & $78(41.1)$ & \\
\hline \multirow[t]{2}{*}{ Tumor grade } & por \& muc & $4(4.2)$ & $13(13.7)$ & $17(8.9)$ & 0.0222 \\
\hline & tub & $91(95.8)$ & $82(86.3)$ & $173(91.1)$ & \\
\hline \multirow[t]{2}{*}{ Lymphatic invasion } & Negative & $29(30.5)$ & $26(27.4)$ & $55(28.9)$ & 0.6313 \\
\hline & Positive & $66(69.5)$ & $69(72.6)$ & $135(71.1)$ & \\
\hline \multirow[t]{2}{*}{ Vascular invasion } & Negative & $28(29.5)$ & $23(24.2)$ & $51(26.8)$ & 0.4130 \\
\hline & Positive & $67(70.5)$ & $72(75.8)$ & $139(73.2)$ & \\
\hline \multirow[t]{3}{*}{ N stage } & N1 & $39(41.1)$ & $36(37.9)$ & $75(39.5)$ & 0.0940 \\
\hline & N2 & $54(56.8)$ & $50(52.6)$ & $104(54.7)$ & \\
\hline & N3 & $2(2.1)$ & $9(9.5)$ & $11(5.8)$ & \\
\hline \multirow{2}{*}{$\begin{array}{l}\text { Number of resected lymph } \\
\text { nodes }\end{array}$} & $<12$ & $5(5.3)$ & 15 (15.8) & $20(10.5)$ & 0.0181 \\
\hline & $\geq 12$ & $90(94.7)$ & $80(84.2)$ & $170(89.5)$ & \\
\hline
\end{tabular}




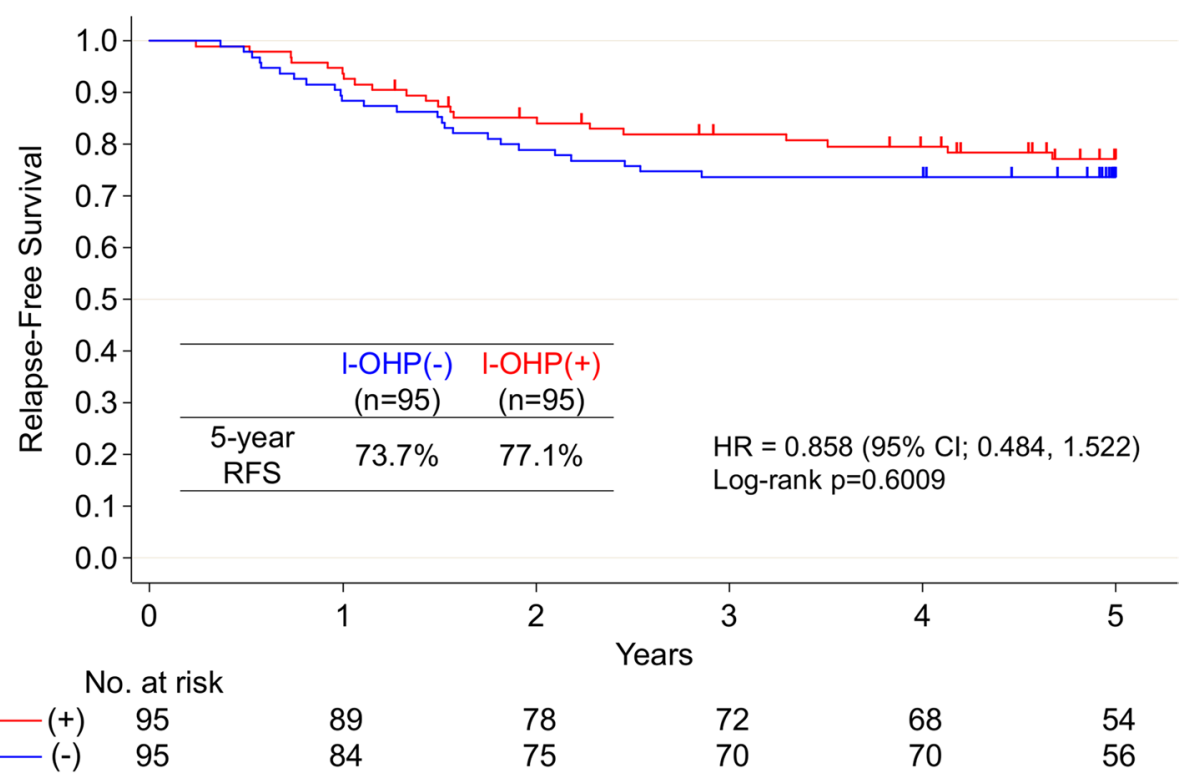

Fig. 2 Five-year relapse-free survival (RFS) curves of all patients treated with [l-OHP(+), indicated in red] and without oxaliplatin [l-OHP(-), indicated in blue]

\section{5-gene classifier subtype analysis}

In the total cohort, 55GC analysis revealed 52 (27.3\%) patients with an MSI-like subtype, 78 (41.1\%) patients with a CIN-like subtype, and $60(31.6 \%)$ patients with a stromal subtype. The clinicopathological characteristics of each subtype are shown in Table 2. MSI-like subtype tumors were more likely to be right-sided, whereas CIN-like subtype tumors were more likely to be leftsided compared to the overall cohort. The MSI-like subtype tumors had a higher proportion of mucinous subtype (6/52 tumors, $11.5 \%)$ compared with CIN-like (0/78 tumors, $0 \%)$ and stromal-like (2/60 tumors, $3.3 \%)$ tumors. We found no difference in lymphatic and vascular invasion between the three subtypes. However, higher proportions of N2/N3 (39/52 tumors, 75.0\%) in MSI-like tumors were found compared to the other subtypes.

\section{Survival analysis according to the 55-gene classifier analysis and chemotherapy regimen}

Comparisons of RFS in patients treated with and without oxaliplatin according to the 55GC subtype are shown in Fig. 3. The 5-year RFS rates were 84.3 and $72.0 \%$ in patients treated with and without oxaliplatin, respectively, for the MSI-like subtype (HR, 0.495; 95\% CI, 0.145-1.692); however, the trend was not statistically significant (log-rank $P=0.25$ ). There was no difference in RFS in CIN-like subtype patients according to oxaliplatin treatment status (HR, 1.241; 95\% CI, 0.465-3.308; $\log$-rank $P=0.67)$. RFS was also unchanged in the stromal subtype patients regardless of oxaliplatin treatment
(HR, 0.791; 95\% CI, 0.329-1.901; log-rank $P=0.60$ ). Further subdivision into left- and right-sided primary cancer of the subtypes showed improved RFS only for left-sided primary cancer of the MSI-like subtype treated with oxaliplatin (Fig. 4; log-rank $P=0.0071$ ). The 5-year RFS rates for the MSI-like subtype in left-sided cancer were 100 and $53.9 \%$ with and without oxaliplatin, respectively. No significant differences in RFS were noted between subtypes when stratified by treatment with (log-rank $P=0.23$ ) and without oxaliplatin (log-rank $P=0.37$; Additional file 1).

Subgroup analysis is shown in Additional file 2. Comparison of RFS in patients treated with or without oxaliplatin revealed no significant differences according to patient characteristics (age, sex) or histopathological findings (tumor location, $\mathrm{T}$ stage, $\mathrm{N}$ stage, tumor grade, and vascular invasion).

\section{Discussion}

Despite a significant progress in the development of prognostic and predictive biomarkers for $\mathrm{CC}$, particularly the $R A S$ mutation status and deficient mismatch repair (dMMR) status to guide therapy for a metastatic disease $[8,9]$, there is an ongoing need for better tools enabling molecular analysis of early stage $\mathrm{CC}$ to guide adjuvant therapy. While dMMR status may indicate a lack of efficacy of fluoropyrimidine-only regimens without oxaliplatin, there is a lack of validated predictive tumor biomarkers for early stage CC $[10,11]$. Several multigene expression profiling systems, such as 
Table 2 Patient characteristics by tumor subtype

\begin{tabular}{|c|c|c|c|c|c|c|}
\hline Factors & & $\begin{array}{l}\text { MSI-like } \\
(N=52) \text { n (\%) }\end{array}$ & $\begin{array}{l}\text { CIN-like } \\
(N=78) \text { n (\%) }\end{array}$ & $\begin{array}{l}\text { Stromal } \\
(N=60) \text { n (\%) }\end{array}$ & Total $(N=190)$ n (\%) & $P$ value \\
\hline \multirow[t]{2}{*}{ Sex } & Male & $28(53.8)$ & $39(50.0)$ & $31(51.7)$ & 98 (51.6) & $p=0.9116$ \\
\hline & Female & $24(46.2)$ & $39(50.0)$ & $29(48.3)$ & $92(48.4)$ & \\
\hline \multirow[t]{2}{*}{ Age } & $<70$ & $37(71.2)$ & $65(83.3)$ & $44(73.3)$ & $146(76.8)$ & $p=0.2011$ \\
\hline & $\geq 70$ & $15(28.8)$ & $13(16.7)$ & $16(26.7)$ & $44(23.2)$ & \\
\hline \multirow[t]{3}{*}{ CEA } & $<U L N$ & $33(63.5)$ & $51(65.4)$ & $29(48.3)$ & $113(59.5)$ & $p=0.1847$ \\
\hline & $\geq U L N$ & $14(26.9)$ & $17(21.8)$ & $20(33.3)$ & $51(26.8)$ & \\
\hline & Unknown & $5(9.6)$ & $10(12.8)$ & $11(18.3)$ & $26(13.7)$ & \\
\hline \multirow[t]{2}{*}{ Tumor location } & Right side & $26(50.0)$ & $18(23.1)$ & $20(33.3)$ & $64(33.7)$ & $p=0.0063$ \\
\hline & Left side & $26(50.0)$ & $60(76.9)$ & $40(66.7)$ & $126(66.3)$ & \\
\hline \multirow[t]{2}{*}{ T stage } & T1-T3 & $30(57.7)$ & $50(64.1)$ & $32(53.3)$ & $112(58.9)$ & $p=0.4335$ \\
\hline & T4 & $22(42.3)$ & $28(35.9)$ & $28(46.7)$ & $78(41.1)$ & \\
\hline \multirow[t]{2}{*}{ Histology } & Non muc & $46(88.5)$ & $78(100.0)$ & $58(96.7)$ & $182(95.8)$ & $p=0.0036$ \\
\hline & muc & $6(11.5)$ & $0(0.0)$ & $2(3.3)$ & $8(4.2)$ & \\
\hline \multirow[t]{2}{*}{ Tumor grade } & por \& muc & $11(21.2)$ & $2(2.6)$ & $4(6.7)$ & $17(8.9)$ & $p=0.0010$ \\
\hline & tub & $41(78.8)$ & 76 (97.4) & $56(93.3)$ & $173(91.1)$ & \\
\hline \multirow[t]{2}{*}{ Lymphatic invasion } & Negative & $14(26.9)$ & $28(35.9)$ & $13(21.7)$ & $55(28.9)$ & $p=0.1754$ \\
\hline & Positive & $38(73.1)$ & $50(64.1)$ & $47(78.3)$ & $135(71.1)$ & \\
\hline \multirow[t]{2}{*}{ Vascular invasion } & Negative & $14(26.9)$ & $26(33.3)$ & $11(18.3)$ & $51(26.8)$ & $p=0.1433$ \\
\hline & Positive & $38(73.1)$ & $52(66.7)$ & $49(89.7)$ & $139(73.2)$ & \\
\hline \multirow[t]{3}{*}{ N stage } & N1 & $13(25.0)$ & $39(50.0)$ & $23(38.3)$ & $75(39.5)$ & $p=0.0021$ \\
\hline & $\mathrm{N} 2$ & $34(65.4)$ & $39(50.0)$ & $31(51.7)$ & $104(54.7)$ & \\
\hline & N3 & $5(9.6)$ & $0(0.0)$ & $6(10.0)$ & $11(5.8)$ & \\
\hline \multirow[t]{2}{*}{ Number of resected lymph nodes } & $<12$ & $3(5.8)$ & $11(14.1)$ & $6(10.0)$ & $20(10.5)$ & $p=0.3125$ \\
\hline & $\geq 12$ & $49(94.2)$ & $67(85.9)$ & $54(90.0)$ & $170(89.5)$ & \\
\hline \multirow[t]{2}{*}{ Adjuvant chemotherapy } & With oxaliplatin & $25(48.1)$ & $42(53.8)$ & $28(46.7)$ & $95(50.0)$ & $p=0.6686$ \\
\hline & Without oxaliplatin & $27(51.9)$ & $36(46.2)$ & $32(53.3)$ & $95(50.0)$ & \\
\hline
\end{tabular}

CEA Carcinoembryonic antigen; ULN Upper limit of normal; muc Mucinous; por \& muc Poorly differentiated and mucinous adenocarcinoma; tub Tubular adenocarcinoma

Oncotype DX (Genomic Health, Redwood City, CA) and ColoPrint (Agendia; Amsterdam, Netherlands), have been developed [12]. However, they are not subtyping systems; therefore, they have a prognostic but no predictive value for chemosensitivity. In contrast, the predictive potential of molecular subtypes in $\mathrm{CC}$ has recently been demonstrated in prospective trials [13-15]. While the classification of CMS is considered the most robust classification based on comprehensive gene expression profiling [5], other classifications have been developed and validated [16-19]. However, optimal methods of subtype identifications and difficulties in the practical widespread measurement of these genotypes in routine clinical practice are the subject of a heated debate [20]. Recently, other gene set classifiers obtained from a microarray analysis used in CMS have been reported. A similar prognostic utility was shown using 99 or 200 gene sets [21]. In this study, we present a potential utility of a 55 gene set, especially when it is accompanied by the assessment of other cancer properties, such as sidedness. The method may provide a prognostic and predictive information for guiding adjuvant therapy in early stage $\mathrm{CC}$ after curativeintent resection.

Post-hoc analyses of tumor tissue from patients in large randomized trials of adjuvant chemotherapy have revealed the overall poor prognosis of certain molecular subtypes. For example, analysis of the National Surgical Adjuvant Breast and Bowel Project (NSABP) C-07 clinical trial demonstrated poor prognosis in both stage II and III patients with a 'stem-like' subtype identified from three different subtyping methods [22]. This is consistent with our findings, which show a tendency for poorer prognosis of the stromal subtype in stage III patients, as well as our previous results regarding stage II/III CC patients [6]. Furthermore, in the aforementioned NSABP $\mathrm{C}-07$ retrospective analysis, the stem-like subtype from the Colorectal Cancer Assigner classification (CRCA) predicted a lack of benefit for the addition of oxaliplatin [22]. Similar to the NSABP C-07 study, our cohort demonstrated a $3.4 \%$ improvement in 5-year RFS due to the 
MSI-like, $n=52$

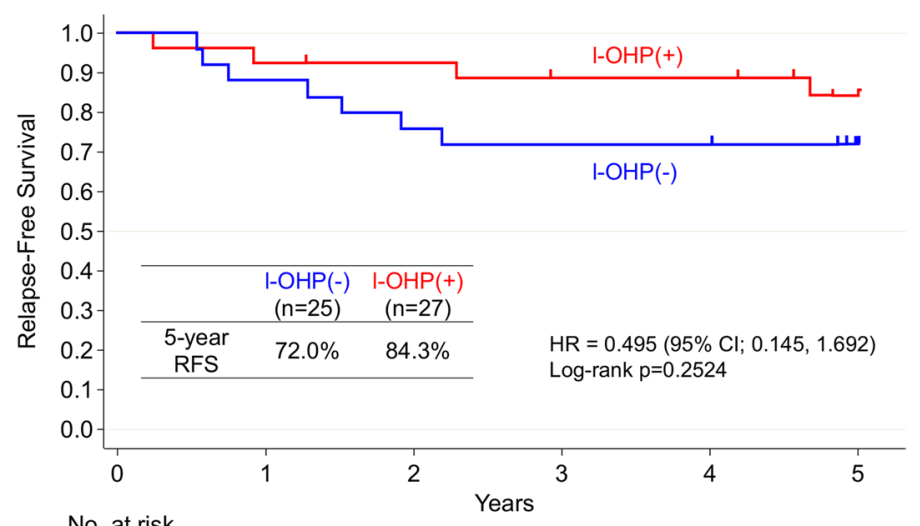

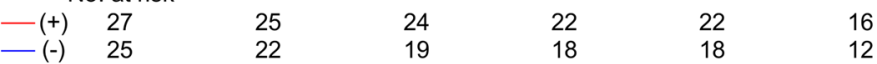

CIN-like, $n=78$

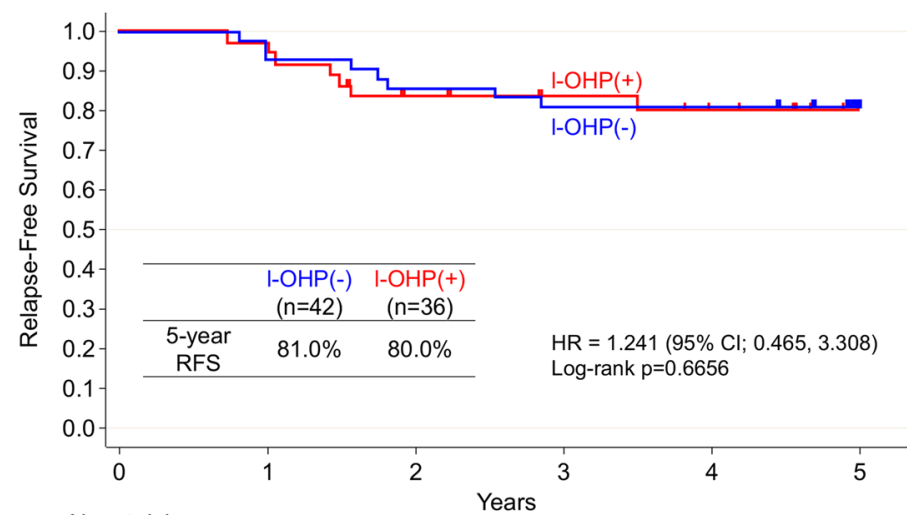

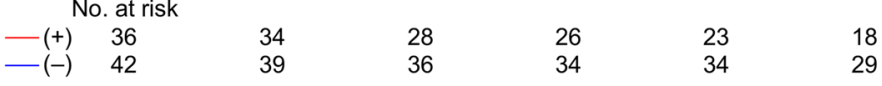

Stromal, $n=60$

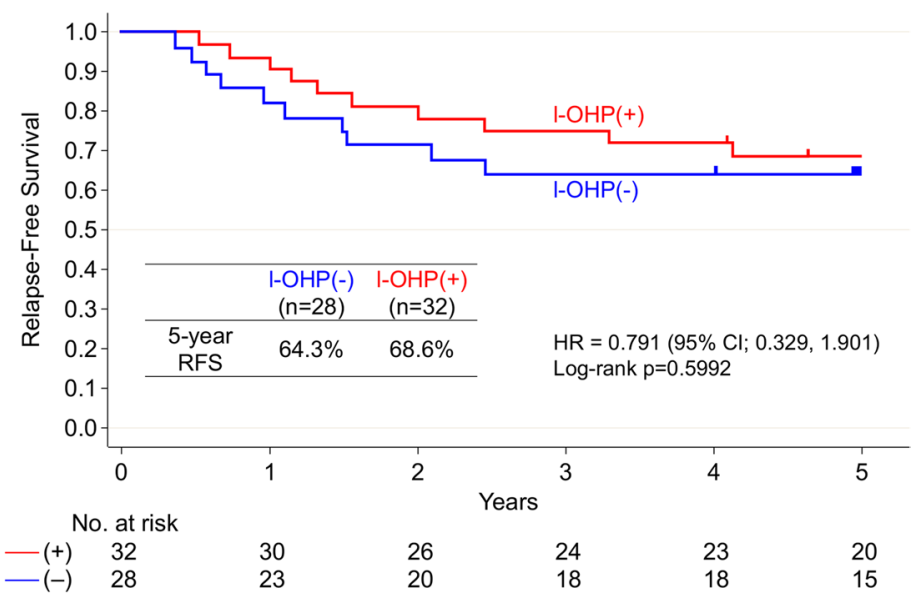

Fig. 3 Five-year relapse-free survival (RFS) curves of patients treated with [l-OHP(+), indicated in red] and without oxaliplatin [l-OHP(-), indicated in blue] according to the 55-gene classifier (55GC) subtypes [top: microsatellite instability (MSI)-like; middle: chromosomal instability (CIN)-like; bottom: stromal] 

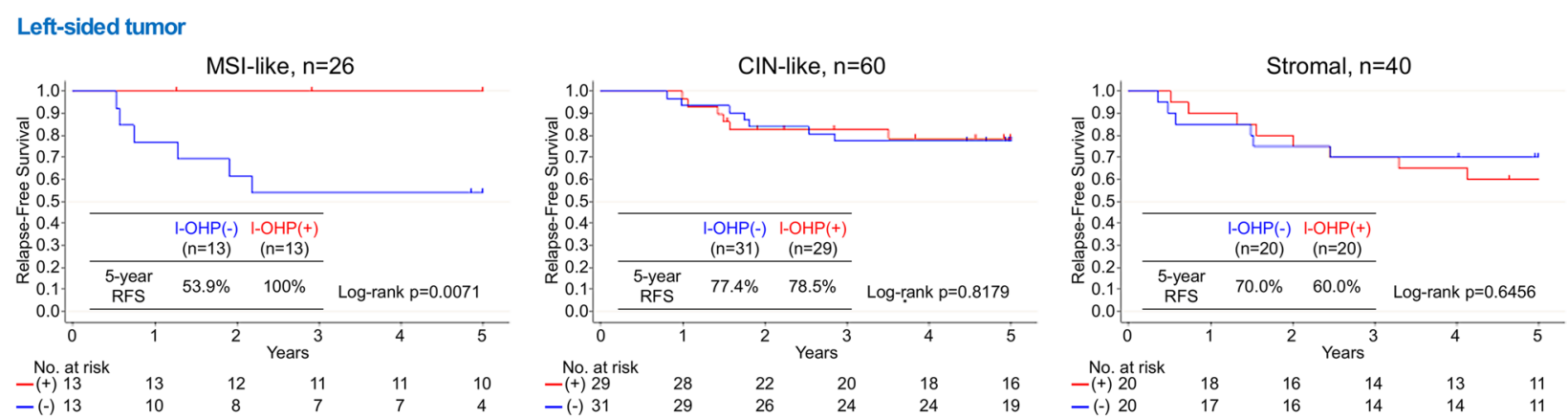

Right-sided tumor
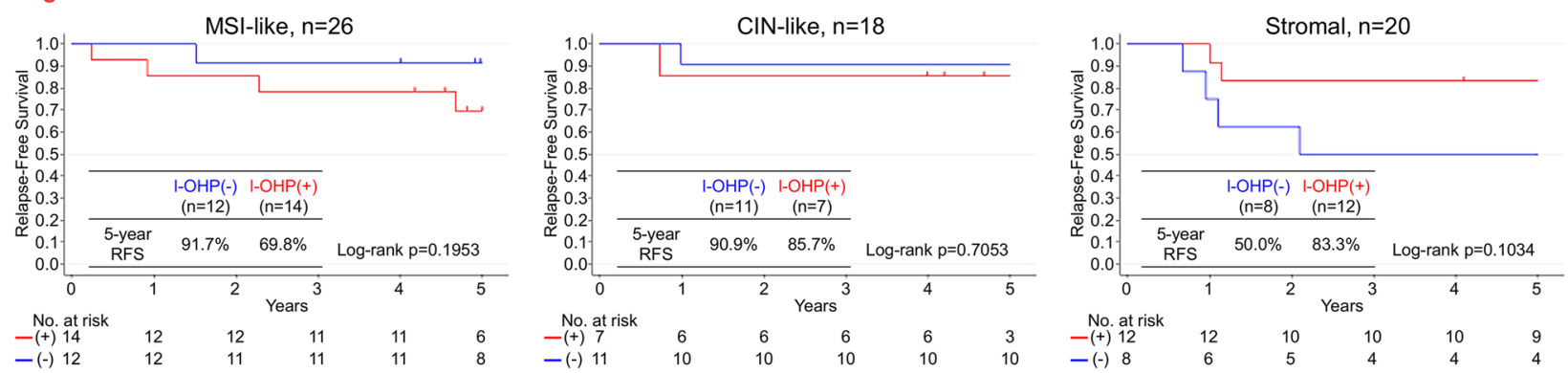

Fig. 4 Five-year relapse-free survival (RFS) curves of patients treated with [l-OHP(+), indicated in red] and without oxaliplatin [l-OHP(-), indicated in blue] according to the 55-gene classifier (55GC) subtypes and primary tumor sidedness (top: left-side; bottom: right-side)

addition of oxaliplatin to adjuvant chemotherapy. Considering this observation, the potential additive benefit of oxaliplatin for adjuvant chemotherapy in the MSI-like subtype from our 55GC system requires further investigation and validation. Similar to the CMS classification, the 55GC system requires caution; the MSI-like subtype is not identical to the MSI-high or dMMR CC. In our cohort, MSI-like subtype tumors had a higher proportion of mucinous tumors and higher proportions of N2/N3 cases compared to the other subtypes. Previous reports have shown that MSI-H tumors have a higher proportion of mucinous subtypes and a lower proportion of lymph node metastasis [23, 24]. One of the reasons why the MSI-H subtype tends to be associated with good prognosis is that this subtype rarely involves lymph node metastasis. Our data showed that the MSI-H like subtype is quite different from the MSI-H and MMR subtypes.

Similar to the CMS classification, our classification showed the potential additive benefit of oxaliplatin for adjuvant chemotherapy in the MSI-like subtype. Recently, circulating tumor DNA (ctDNA) has been shown to be a promising and accurate predictive marker for tumor recurrence $[25,26]$. Treatment of ctDNA-positive patients with aggressive chemotherapy may therefore reduce recurrence rates. In line, we initiated a nationwide large-scale clinical trial named CIRCULATE-Japan [27], which consists of a prospective observational study and two accompanying interventional studies to elucidate the predictive value of ctDNA for the recurrence risk. ctDNA may be a strong predictive marker for recurrence; however, it could not reveal the intrinsic subtype of cancer. Therefore, an optimized combination of a few prognostic methods will probably be utilized in the clinic in the future.

The major limitation of the current study is the relatively small number of patients in each analyzed subgroup, especially after accounting for propensity score matching. Nevertheless, the results suggest the potential benefit of adjuvant chemotherapy with oxaliplatin in the MSI-like cancer subtype when accounting for tumor sidedness. These findings require further prospective validation in an independent cohort to determine their true clinical significance. Combined with deeper analysis of genomic and histopathologic correlates, including the immune infiltrate and tumor microenvironment, our data could improve our understanding of the biological underpinnings of each tumor subtype, resulting in more accurate diagnosis and treatment stratification.

\section{Conclusions}

In conclusion, the current 55GC study highlights that oxaliplatin may have an additive effect in adjuvant chemotherapy for the MSI-like CC subtype, especially for left-sided primary tumors. Hence, future studies with 
larger numbers of CC cases are warranted to validate our findings.

\begin{abstract}
Abbreviations
55GC: 55 gene classifier; CC: Colon cancer; CEA: Carcinoembryonic antigen; Cl: Confidence interval; CIN: Chromosomal instability; CMS: Consensus molecular subtypes; CONSORT: Consolidated Standards of Reporting Trials; CRCA: Colorectal Cancer Assigner; DNA: Deoxyribonucleic acid; FFPE: Formalin-fixed paraffin-embedded; HR: Hazard ratio; MSI: Microsatellite instability; NSABP: National Surgical Adjuvant Breast and Bowel Project; P: P-value; RFS: Relapsefree survival; RNA: Ribonucleic acid; STAR: Stratification recurrence; ULN: Upper limit of normal; UMIN: University Hospital Medical Education Network.
\end{abstract}

\section{Supplementary Information}

The online version contains supplementary material available at https://doi. org/10.1186/s12885-021-09088-6.

Additional file 1: Figure S1. Five-year relapse-free survival (RFS) curves in the 55-gene classifier (55GC) subtypes according to the adjuvant chemotherapy received [blue: microsatellite instability (MSI)-like; red: chromosomal instability (CIN)-like; green: stromal].

Additional file 2: Figure S2. Subgroup analysis.

\section{Acknowledgments}

Editorial support, in the form of medical writing, assembling tables, and creating high-resolution images based on authors' detailed directions, collating author comments, copyediting, fact-checking, and referencing, was provided by Editage, Cactus Communications, and was funded by Sysmex Corporation.

\section{Authors' contributions}

S.T. and T.M.: quality control of data and algorithms. E.O., E.S., M.S., S.T., T.M., and K.H.: data analysis and interpretation. M.S.: statistical analysis. E.O., E.S., M.S., S.T., T.M., and K.H.: manuscript preparation. E.O., E.S., S.Y., M.I., S.H., Y.T., H.I., T.K., M.M., N.T., Ma.S., M.T., H.O., Y.H., S.O., S.T., T.M., K.H.: study conceptualization, study design, data acquisition, manuscript editing, and manuscript review. The author(s) read and approved the final manuscript.

\section{Funding}

This work was supported by the Sysmex Corporation. The funding source was not involved in study design; in the collection, analysis, or interpretation of data; in the writing of the report; or in the decision to submit the article for publication.

\section{Availability of data and materials}

The cDNA microarray datasets generated during the current study are available from the corresponding author upon a reasonable request. The data were not reposited in the Gene Expression Omnibus (GEO) database due to concerns related to the Japanese Act of the Protection of Personal Information. guidelines and regulations. Because this study was a retrospective observational study carried out in Japan, informed consent was obtained using the opt-out/opt-in approach according to each participating institution's policy (as per Japanese ethical guidelines for an observational study, consent of the family is not required for dead participants).

\section{Consent for publication}

Not applicable.

\section{Competing interests}

E.O. has been part of a speaker's bureau at Bayer, Chugai Pharmaceutical, Eli Lilly, Merck Serono, Ono Pharmaceutical, Taiho Pharmaceutical, Takeda Pharmaceutical, and Yakult Honsha. M.S. has a consulting/advisory role at Sysmex. S.Y. has been part of a speaker's bureau at Chugai Pharmaceutical, Covidien, Eli Lilly, Merck Serono, Johnson \& Johnson, and Taiho Pharmaceutical. M.I. has received honoraria from Merck Serono, Taiho Pharmaceutical, and Yakult Honsha, and has a consulting/advisory role at Taiho Pharmaceutical and Yakult Honsha. N.T. has received research funding from Chugai Pharmaceutical and Taiho Pharmaceutical. Y.H. has received honoraria from Bayer, Chugai Pharmaceutical, Kaken Pharmaceutical, Merck Serono, Sanofi, Taiho Pharmaceutical, and Takeda Pharmaceutical, and has received research funding from Chugai Pharmaceutical, Kaken Pharmaceutical, Merck Serono, Sanofi, Taiho Pharmaceutical, and Takeda Pharmaceutical. S.T. and T.M. are employed by Sysmex and own stock shares of Sysmex. E.S., S.H., Y.T., H.I., T.K., M. M, M.S., M.T., H.O., S.O., and K.H. declare no conflicts of interest.

\section{Author details}

${ }^{1}$ Department of Surgery and Science, Graduate School of Medical Sciences, Kyushu University, 3-1-1, Maidashi, Higashi-ku, Fukuoka 812-8582, Japan. ${ }^{2}$ Department of Surgery, National Defense Medical College, Tokorozawa, Japan. ${ }^{3} \mathrm{Cl}$ inical Research Institute, National Hospital Organization Kyushu Cancer Center, Fukuoka, Japan. ${ }^{4}$ Department of Gastroenterological Surgery, Saitama Medical University International Medical Center, Hidaka, Japan. ${ }^{5}$ Department of Translational Oncology, Graduate School of Medical and Dental Science, Tokyo Medical and Dental University, Tokyo, Japan. ${ }^{6}$ Department of Surgery, Saiseikai Yokohamashi Nanbu Hospital, Yokohama, Japan. ${ }^{7}$ Department of Gastroenterological Surgery, Niigata Cancer Center Hospital, Niigata, Japan. ${ }^{8}$ Department of Digestive Tract and General Surgery, Saitama Medical Center, Saitama Medical University, Kawagoe, Japan. ${ }^{9}$ Department of Gastroenterological Surgery, Clinical Research Center, Cancer Research Division, National Hospital Organization Kyushu Medical Center, Fukuoka, Japan.

${ }^{10}$ Gastroenterological Surgery, National Hospital Organization Kyushu Cancer Center, Fukuoka, Japan. "'Department of Surgery, Hyogo College of Medicine, Nishinomiya, Japan. ${ }^{12}$ Colorectal Surgery Division, Kanagawa Cancer Center, Yokohama, Japan. ${ }^{13}$ Coloproctology Center, Takano Hospital, Kumamoto, Japan. ${ }^{14}$ Department of Colorectal Surgery, Tochigi Cancer Center, Utsunomiya, Japan. ${ }^{15}$ Department of Surgery, Teikyo University School of Medicine, Tokyo, Japan. ${ }^{16}$ Department of Surgery, Tohoku University Hospital, Sendai, Japan. ${ }^{17}$ LS Business, Sysmex Corporation, Kobe, Japan.

Received: 1 June 2021 Accepted: 18 November 2021

Published online: 14 December 2021

\section{Declarations}

\section{Ethics approval and consent to participate}

The study protocol was approved by the following institutional review boards: Kyushu University (study ID 28-69), National Defense Medical College (study ID 2477), Saitama Medical University International Medical Center (study ID 16-051), Tokyo Medical and Dental University (study ID G2016-007), Saiseikai Yokohamashi Nanbu Hospital (study ID 2017-D21), Niigata Cancer Center Hospital (study ID 796), Saitama Medical University Saitama Medical Center (study ID 1812), National Hospital Organization Kyushu Medical Center (study ID 16C058), National Hospital Organization Kyushu Cancer Center (study ID 2016-48), Hyogo College of Medicine (study ID Hi326), Kanagawa Cancer Center (study ID 2017-8), Takano Hospital (study ID 16-04), Tochigi Cancer Center (study ID A432), Teikyo University School of Medicine (study ID 16-057), Tohoku University (study ID 2016-1-222) and Sysmex Corporation (study ID 2015-71). All procedures were performed in accordance with the relevant

\section{References}

1. Arnold M, Sierra MS, Laversanne M, Soerjomataram I, Jemal A, Bray F. Global patterns and trends in colorectal cancer incidence and mortality. Gut. 2017;66(4):683-91.

2. André T, Boni C, Navarro M, Tabernero J, Hickish T, Topham C, et al. Improved overall survival with oxaliplatin, fluorouracil, and leucovorin as adjuvant treatment in stage II or III colon cancer in the MOSAIC trial. J Clin Oncol. 2009;27(19):3109-16.

3. Grothey A, Sobrero AF, Shields AF, Yoshino T, Paul J, Taieb J, et al. Duration of adjuvant chemotherapy for stage III colon cancer. N Engl J Med. 2018;378(13):1177-88.

4. Gunderson LL, Jessup JM, Sargent DJ, Greene FL, Stewart AK. Revised TN categorization for colon cancer based on national survival outcomes data. J Clin Oncol. 2010;28(2):264-71. 
5. Guinney J, Dienstmann R, Wang X, de Reyniès A, Schlicker A, Soneson C, et al. The consensus molecular subtypes of colorectal cancer. Nat Med. 2015;21(11):1350-6.

6. Gotoh K, Shinto E, Yoshida Y, Ueno H, Kajiwara Y, Yamadera M, et al. Prognostic model of stage II/III colon cancer constructed using gene expression subtypes and KRAS mutation status. J Clin Exp Oncol. 2018;07(02). Available from: https://doi.org/10.4172/2324-9110.1000214.

7. Shinto E, Oki E, Shimokawa M, Yamaguchi S, Ishiguro M, Morita M, et al. A validation study for recurrence risk stratification of stage II colon cancer using the 55-gene classifier. Oncology. 2020;98(8):534-41.

8. Karapetis CS, Khambata-Ford S, Jonker DJ, O'Callaghan CJ, Tu D, Tebbutt $\mathrm{NC}$, et al. K-ras mutations and benefit from cetuximab in advanced colorectal cancer. N Engl J Med. 2008;359(17):1757-65.

9. Le DT, Durham JN, Smith KN, Wang H, Bartlett BR, Aulakh LK, et al. Mismatch repair deficiency predicts response of solid tumors to PD-1 blockade. Science. 2017;357(6349):409-13.

10. Sargent DJ, Marsoni S, Monges G, Thibodeau SN, Labianca R, Hamilton SR, et al. Defective mismatch repair as a predictive marker for lack of efficacy of fluorouracil-based adjuvant therapy in colon cancer. J Clin Oncol. 2010;28(20):3219-26.

11. Koncina E, Haan S, Rauh S, Letellier E. Prognostic and predictive molecular biomarkers for colorectal Cancer: updates and challenges. Cancers (Basel). 2020;12(2):319.

12. Sharif $\mathrm{S}, \mathrm{O}^{\prime} \mathrm{C}$ onnell MJ. Gene signatures in stage II colon cancer: a clinical review. Curr Colorectal Cancer Rep. 2012;8(3):225-31.

13. Mooi JK, Wirapati P, Asher R, Lee CK, Savas P, Price TJ, et al. The prognostic impact of consensus molecular subtypes (CMS) and its predictive effects for bevacizumab benefit in metastatic colorectal cancer: molecular analysis of the AGITG MAX clinical trial. Ann Oncol. 2018;29(11):2240-6.

14. Lenz H-J, Ou F-S, Venook AP, Hochster HS, Niedzwiecki D, Goldberg RM, et al. Impact of consensus molecular subtype on survival in patients with metastatic colorectal cancer: Results from CALGB/SWOG 80405 (alliance). J Clin Oncol. 2019;37(22):1876-85.

15. Stintzing S, Wirapati $P$, Lenz H-J, Neureiter D, Fischer von Weikersthal L, Decker T, et al. Consensus molecular subgroups (CMS) of colorectal cancer (CRC) and first-line efficacy of FOLFIRI plus cetuximab or bevacizumab in the FIRE3 (AIO KRK-0306) trial. Ann Oncol. 2019;30(11):1796-803.

16. Perez-Villamil B, Romera-Lopez A, Hernandez-Prieto S, Lopez-Campos G, Calles A, Lopez-Asenjo J-A, et al. Colon cancer molecular subtypes identified by expression profiling and associated to stroma, mucinous type and different clinical behavior. BMC Cancer. 2012;12(1):260.

17. Oh SC, Park Y-Y, Park ES, Lim JY, Kim SM, Kim S-B, et al. Prognostic gene expression signature associated with two molecularly distinct subtypes of colorectal cancer. Gut. 2012;61(9):1291-8.

18. Sadanandam A, Lyssiotis CA, Homicsko K, Collisson EA, Gibb WJ, Wullschleger $\mathrm{S}$, et al. A colorectal cancer classification system that associates cellular phenotype and responses to therapy. Nat Med. 2013:19(5):619-25.

19. De Sousa E, Melo F, Wang X, Jansen M, Fessler E, Trinh A, et al. Poorprognosis colon cancer is defined by a molecularly distinct subtype and develops from serrated precursor lesions. Nat Med. 2013;19(5):614-8.

20. Fontana E, Eason K, Cervantes A, Salazar R, Sadanandam A. Context matters-consensus molecular subtypes of colorectal cancer as biomarkers for clinical trials. Ann Oncol. 2019;30(4):520-7.

21. Morris JS, Luthra R, Liu Y, Duose DY, Lee W, Reddy NG, et al. Development and validation of a gene signature classifier for consensus molecular subtyping of colorectal carcinoma in a CLIA-certified setting. Clin Cancer Res. 2021;27(1):120-30.

22. Song N, Pogue-Geile KL, Gavin PG, Yothers G, Kim SR, Johnson NL, et al. Clinical outcome from oxaliplatin treatment in stage II/III colon cancer according to intrinsic subtypes: secondary analysis of NSABP C-07/NRG oncology randomized clinical trial: secondary analysis of NSABP C-07/ NRG oncology randomized clinical trial. JAMA Oncol. 2016;2(9):1162-9.

23. Kim J, Hong Y, Kim H, Kim K, Kim S, Lim S, et al. Microsatellite instability was not associated with survival in stage III Colon Cancer treated with adjuvant chemotherapy of Oxaliplatin and Infusional 5-fluorouracil and Leucovorin (FOLFOX). Ann Surg Oncol. 2016;24(5):1289-94.

24. Nakaji Y, Oki E, Nakanishi R, Ando K, Sugiyama M, Nakashima Y, et al. Prognostic value of BRAF V600E mutation and microsatellite instability in Japanese patients with sporadic colorectal cancer. J Cancer Res Clin Oncol. 2016;143(1):151-60.

25. Reinert T, Schøler L, Thomsen R, Tobiasen H, Vang S, Nordentoft I, et al Analysis of circulating tumour DNA to monitor disease burden following colorectal cancer surgery. Gut. 2015;65(4):625-34.

26. Tie J, Wang Y, Tomasetti C, Li L, Springer S, Kinde I, et al. Circulating tumor DNA analysis detects minimal residual disease and predicts recurrence in patients with stage II colon cancer. Sci Transl Med. 2016;8(346):346ra92.

27. Taniguchi H, Nakamura Y, Kotani D, Yukami H, Mishima S, Sawada K, et al. CIRCULATE-Japan: circulating tumor DNA-guided adaptive platform trials to refine adjuvant therapy for colorectal cancer. Cancer Sci. 2021;1(10):14926.

\section{Publisher's Note}

Springer Nature remains neutral with regard to jurisdictional claims in published maps and institutional affiliations.
Ready to submit your research? Choose BMC and benefit from:

- fast, convenient online submission

- thorough peer review by experienced researchers in your field

- rapid publication on acceptance

- support for research data, including large and complex data types

- gold Open Access which fosters wider collaboration and increased citations

- maximum visibility for your research: over $100 \mathrm{M}$ website views per year

At BMC, research is always in progress.

Learn more biomedcentral.com/submissions 